\title{
Flux heterogeneity through incidence angle and particle energy in steering-enhanced growth
}

\author{
Herbert Wormeester* and Bene Poelsema \\ MESA + Research Institute, University of Twente, P.O. Box 217, 7500 AE Enschede, The Netherlands
}

(Received 22 May 2002; published 7 October 2002)

\begin{abstract}
During growth Van der Waals forces between the incident atoms and the substrate lead to steering with sometimes important implications for the morphology of the molecular-beam-epitaxy- or sputter grown films. Deterministic classical trajectory calculations, modelling the atom-substrate interaction with a Lennard Jones potential, have been run to provide insight in the influence of the polar angle of incidence and the energy of the atoms on steering for 1 and 3 monolayer high islands. For low energies (10-100 meV) substantial flux redistribution has been found. A major part of the flux directed to the ascending steps is transferred on top of the protrusion, while even reversal of the lateral velocity at the descending steps occurs. At grazing incidence strong deviations from geometric shadowing behavior is observed even for energies as high as 1 to $10 \mathrm{eV}$.

DOI: 10.1103/PhysRevB.66.165406

PACS number(s): 68.55.Ac, 81.10.Aj, 81.15.Aa
\end{abstract}

\section{INTRODUCTION}

One of the axioms in long-standing kinetic growth studies is the uniform distribution of the deposited particles when they land on the surface. The influence of the substrate on the particle trajectory was already recognized by Shevchik, ${ }^{1}$ who discussed the influence of the covalent binding potential, the van der Waals potential for neutral incident atoms and the electrostatic potential for charged incident atoms on the particle trajectory. He describes how these interactions for normal incident atoms lead to a roughening of the surface. Long-range attractive forces between incident atoms and substrate atoms were found to lead to substantial acceleration towards the surface, ${ }^{2,3}$ although no transient mobility effects were found resulting from the transformation from potential energy into motion parallel to the substrate during adsorption. Luedtke and Landman ${ }^{4}$ combined molecular dynamics and deflection of trajectories through interactions between substrate and incoming particle for the growth of $a$-Si. Their simulation for normal and $60^{\circ}$ incidence shows a distinct deflection of trajectories. For the $60^{\circ}$ case the deflection of trajectories leads to asymmetric three-dimensional structures that, due to geometric shadowing, in further growth lead to a columnar microstructure. They found that the angle of the column with the respect to the normal corresponds well with the phenomenologically derived tangent rule. However, until recently no clear experimental verification of the influence of the substrate on the incoming particle trajectory has been given. Van Dijken et al. ${ }^{5-8}$ showed for $\mathrm{Cu} / \mathrm{Cu}(001)$ and $\mathrm{Co} /$ $\mathrm{Cu}(001)$ clearly changes in morphology as the result of this effect, which they denoted as steering. Especially for grazing incidence the effect becomes pronounced and explains the observed anisotropy of the three-dimensional (3D) structures on the fourfold symmetric substrate. As already predicted by Shevchik, ${ }^{1}$ the steering effect enhances the formation and growth of surface irregularities, which in turn themselves enhance the distortion of the particle trajectories. Steering features thus autocatalyze the kinetic roughening of the growth front. The recent experimental results have stimulated several groups. A detailed incorporation in a nonlinear stochastic growth equation has been performed recently by Raible et al. ${ }^{9,10}$ A combined molecular dynamics and kinetic
Monte Carlo simulation of the submonolayer growth of $\mathrm{Cu}$ at grazing incidence on $\mathrm{Cu}(001)$ was made by Seo et al. ${ }^{11}$ Montalenti et al. discussed the steering enhanced growth at normal incidence for $\mathrm{Ag} / \mathrm{Ag}(001)$ at very low substrate temperatures $(<70 \mathrm{~K}) .^{12,13}$ They also showed an extensive roughening with decreasing energy of the incident particles. The key parameter for the influence of the substrate on the particle trajectory is the time of the incident atom in the influence sphere of the substrate. From the initial work of van Dijken et al., ${ }^{6}$ it is known that from about $2 \mathrm{~nm}$ above the surface the trajectory of the atom changes. Grazing incidence enhances the length of the trajectory in the influence sphere and thus the time period. However, also the energy of the atoms is of prime importance. For more energetic particles less influence on the trajectory is expected, with a geometric shadowing behavior for the high energy limit. In this paper the influence of the kinetic energy on the trajectory of an incident $\mathrm{Cu}$ atom between 0.01 and $10 \mathrm{eV}$ on a $\mathrm{Cu}(001)$ surface is evaluated for several characteristic situations in the early stages of growth. These kinetic energies are within experimental range by either velocity selection of a thermal evaporation source or sputter deposition. We will show that the size of the islands has only influence for grazing incidence, while even for $10 \mathrm{eV}$ particles an extended shadow region is observed. Both monolayer and three layer high islands are considered.

\section{STEERING}

A substantial acceleration and in the case of grazing incidence deposition a substantial deflection towards the surface can be expected for thermal energy particles. ${ }^{1,3}$ Atom trajectory calculations were performed with the attractive force modeled by a Lennard-Jones $(12,6)$ pairwise potential. The potential is given by

$$
V(R)=D\left(\frac{a_{/ /}}{R}\right)^{6}\left[\left(\frac{a_{/ /}}{R}\right)^{6}-2\right]
$$

where $a_{/ /}$is the nearest neighbor distance $(2.55 \AA$ for $\mathrm{Cu}$ ) and $D$ is a pairwise energy which was fitted to the cohesive energy $(D=0.4093 \mathrm{eV}$ for $\mathrm{Cu})$. Normally such a pairwise 


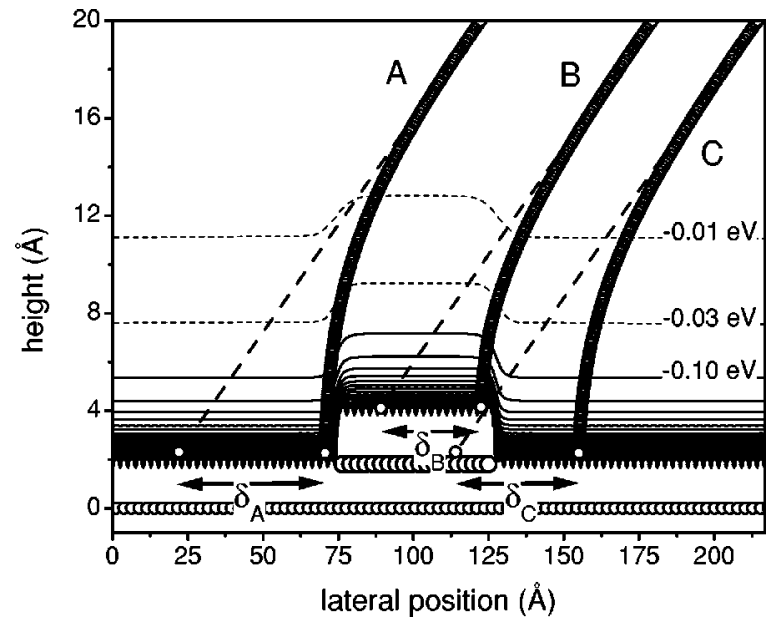

FIG. 1. Calculated equipotential energy contours and three atom trajectories for a surface with a monolayer high island on top of it. The increase in the attractive potential is $-0.1 \mathrm{eV}$ for the solid contour lines. The trajectory calculations for a deposition geometry of 80 starts at $20 \AA$ above the surface.

additive Lennard-Jones potential is believed to be inadequate to describe the attractive potential of metals in detail. ${ }^{14}$ Sanders et al., ${ }^{2}$ however, found that atom trajectory calculations with such a Lennard-Jones potential are satisfactorily close to calculations with their most accurate many body density functional based potential energy surface. The Lennard-Jones pairwise potential was therefore used to estimate the effect of steering.

Figure 1 shows a cross-sectional view through the $\mathrm{Cu}(001)$ substrate along the [110] azimuth. Please note that the vertical scale is expanded by a factor of 8 with respect to the horizontal one. On the substrate a monolayer high adatom island is constructed. The figure exhibits calculated equipotential energy contours (increment $-0.1 \mathrm{eV}$ ) as well as three calculated atom trajectories for atoms deposited at a grazing angle of $80^{\circ}$. The equipotential energy contours show substantial distortion in the attractive potential, which is related to the ascending and descending step edges. The lateral length of the distortion is about $15 \AA$ for the $-0.01 \mathrm{eV}$ contour. Calculations show that $150 \mathrm{meV} \mathrm{Cu}$ atoms, deposited with a grazing angle of incidence of $80^{\circ}$, actually hit the surface at an angle of $17^{\circ}$, when the trajectory passes through an undistorted attractive potential (trajectory $\mathrm{C}$ in Fig. 1). When atoms pass through a distorted attractive potential, however, the trajectories can deviate substantially (trajectories A and B in Fig. 1). The result of a surface roughness induced variation in atom trajectories is illustrated most clearly when the distance between the impact point at which the atoms actually hit the surface and their target point ( $\delta_{\mathrm{A}}, \delta_{\mathrm{B}}$, and $\delta_{\mathrm{C}}$ in Fig. 1$)$ is considered. The target point is the intersection of the asymptotic long distance part of the trajectory with the surface. Atoms following trajectory A in Fig. 1 pass through a distorted attractive potential, which is related to a descending step edge. They experience a lateral force attracting them towards the step edge. As particle A experiences this lateral force a relatively longer time, the value of $\delta_{\mathrm{A}}$ is enhanced with respect

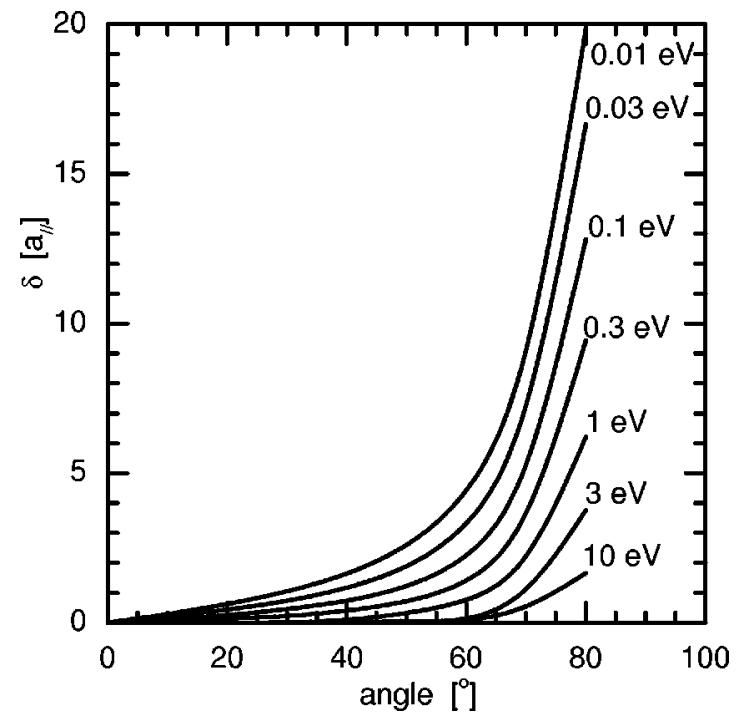

FIG. 2. Energy and incidence angle dependence of the lateral difference in impact position on a flat surface through steering.

to $\delta_{\mathrm{C}}$. On the other hand, atoms following trajectory $\mathrm{B}$ in Fig. 1 suddenly experience the surface at a much smaller distance when they pass through the distorted attractive potential, which is now related to an ascending step edge. Therefore the actual position of impact is much closer to the target point and $\delta_{\mathrm{B}}$ is reduced with respect to $\delta_{\mathrm{C}}$. In addition, the lateral forces between the ascending step edge and the approaching atom reduce $\delta_{\mathrm{B}}$. The consequence is a local enhancement of the incident atom flux just behind the leading edge of the adatom island. In fact all arriving atoms whose trajectories pass through areas of substantial distortion of the attractive potential related to ascending step edges contribute to enhanced flux. They are focused on top of the adatom island, with the maximum atom flux close to the leading edge. This focussing of incident atom flux on top of adatom structures is called steering.

On a flat surface, the action of dispersion forces will not lead to a heterogeneity of the flux, however, it is interesting to see how the shift $\delta$ depends on angle of incidence and energy, see Fig. 2. For very low energies this shift can extend over 20 interatomic distances for $80^{\circ}$ incidence. However, also for the $3 \mathrm{eV}$ particles still a substantial shift of almost 4 lattice units is observed and for grazing incidence, at least particles of $10 \mathrm{eV}$ seem to be needed for the geometric shadow limit, i.e., no influence on the trajectory. Below $50^{\circ}$ angle of incidence, hardly any influence is seen for the relevant energies of a thermal energy atom deposition source. For sake of completeness we note that obviously, during growth, even starting from a flat surface, at some point protrusions will form and are already existing in the form of step edges, so that steering causes an instability in the growth process (see also Refs. 9, and 10)

\section{INFLUENCE OF MONOLAYER ISLANDS}

The flux redistribution was evaluated by calculating trajectories with an equally spaced initial starting grid $20 \AA$ above the surface. A spacing of $a_{/ /} / 100$ was used and the 


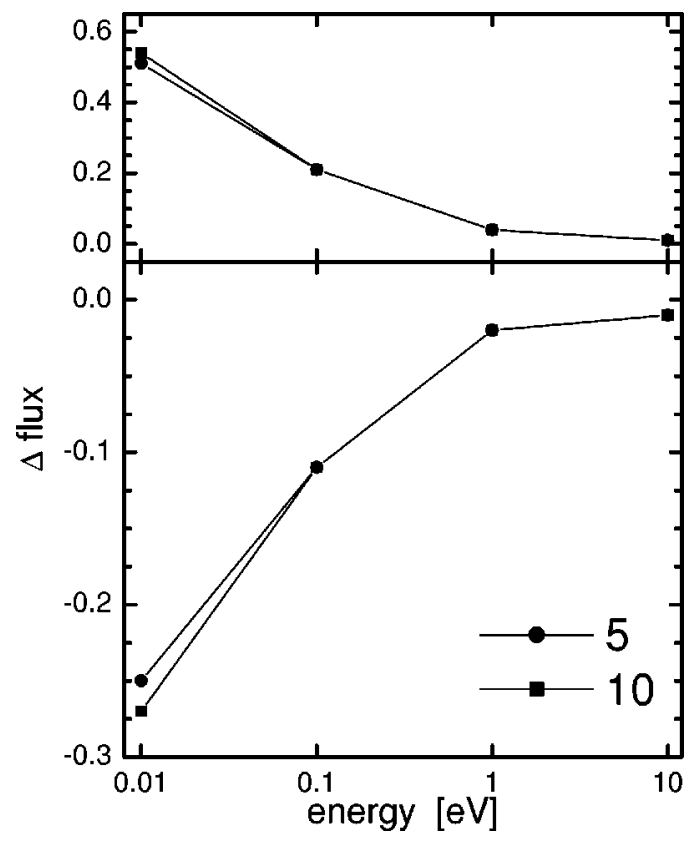

FIG. 3. Flux redistribution for a monolayer island on a $\mathrm{Cu}(001)$ surface as a function of the energy of the incident $\mathrm{Cu}$ atom. The deposition beam is at normal incidence. Shown are the additional flux on top of the island (upper panel) and the additional flux next to the island (lower panel). Shown are calculations for a 5 and 10 atom wide island.

trajectories were calculated until the incoming particle was at $2.3 \AA$ from the nearest atom in the substrate. The lateral position at this height was taken as the final adsorption position. The adsorption positions were discretized with one adsorption position per surface atom. At the step the lower terrace starts with an adsorption position of $1.5 a_{/ /}$. The number of trajectories ended per adsorption position was counted. A total of 5000-8000 trajectories, i.e., 50-80 atomic distances, were sufficient for calculating the heterogeneity in the flux distribution as the result of the modelled disturbance on the substrate. Previously, the flux was calculated from the change in lateral distance between two neighboring atom trajectories. However, this gives large errors at the step edges. The used method assures that the integral of the flux is correct.

The flux redistribution due to a monolayer island with the deposition beam at normal incidence is shown in Fig. 3. In this the integrated additional flux, $\Delta$ flux, on top and at either side of the island is given. The homogeneous incidence flux $\mathrm{F}$ is set at a rate of 1 particle per $a_{/ /}$per unit of time. The additional number of particles per $a_{/ /}$per unit of time on top, in front or behind the island is thus given by $\Delta$ flux $\times F$.

The lateral size of the island has hardly any influence for normal incidence, except for a minor lower additional flux for the smallest island at the lowest particle kinetic energy. Obviously the height of the island does have a substantial influence on steering (see below). We note that lateral dimensions below $\approx 5$ atoms lead to a decrease of steering, depending on the atom energy and polar angle. For the high energy region $E>1 \mathrm{eV}$ hardly any additional flux is observed on top of the island and as a consequence the flux

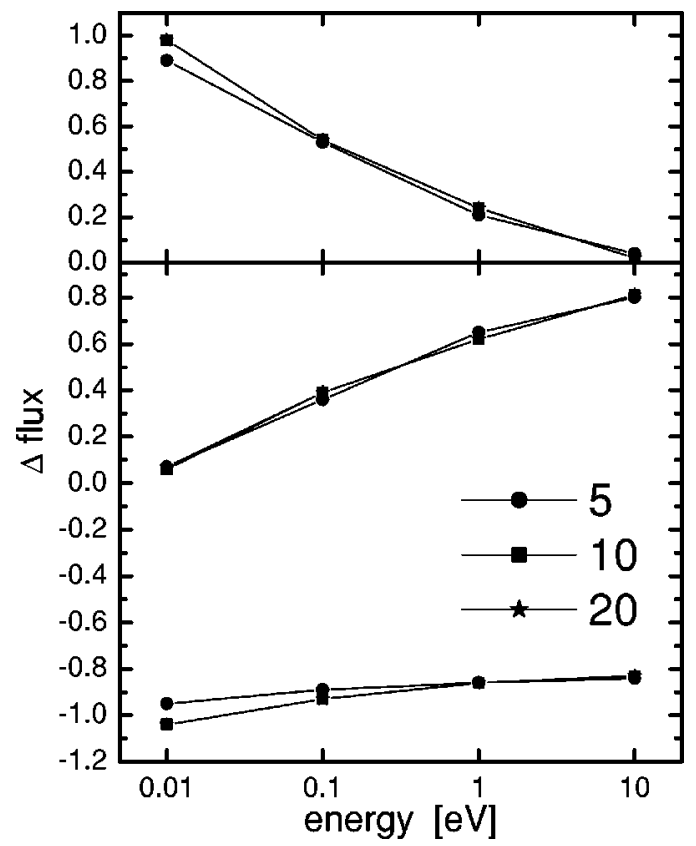

FIG. 4. Flux redistribution for a monolayer island on a $\mathrm{Cu}(001)$ surface as a function of the energy of the incident $\mathrm{Cu}$ atom. The deposition beam is at $50^{\circ}$. Shown are the additional flux on top of the island (upper panel) and the additional flux in front (positive) and behind the island (lower panel). Calculations are depicted for a 5,10 , and 20 atom wide island. The dashed line indicates the geometric shadow value of the decrease in flux behind the island.

next to the island on the terrace is hardly reduced.

Figure 4 shows the situation for a beam with an angle of incidence of $50^{\circ}$. Also in this case the island size has only minor influence on the result, except again a small difference for the lowest energy. In this situation, the negative change in flux on the terrace behind the island is for all energies about equal. In the limit of high energies, the geometric shadow length is the measure for the decrease in flux and indicated by the dashed line. For this incidence angle, the decrease in flux is for all energies comparable to this limiting value. It depends strongly on the energy where this negative flux is deposited, in front or on top of the island. For high energies, the on top flux is hardly effected and the ascending step of the island functions as a shadow barrier for the incident flux. For the growth of the island, this implies that the center of gravity of the island only moves in the direction of the deposition beam while the morphology of the island itself is hardly changed. For low energies, the additional flux is deposited on top of the island. This will result in an additional roughening of the surface, the extent determined by the island size and the presence of a possible Ehrlich-Schwoebel barrier that would decrease interlayer mass transport. ${ }^{11}$ The additional flux on top of the island is the first ingredient towards an anisotropic growth of the island as a much smaller transfer of flux occurs from the edges parallel to the incident beam. The additional flux on top of the island can remain on the island or through interlayer mass transport descent. This mass current will be rather equal for all four sides of the island for the square lattice of a $\mathrm{Cu}(001){ }^{6,11} \mathrm{~A}$ broadening of the island in the direction perpendicular to the 


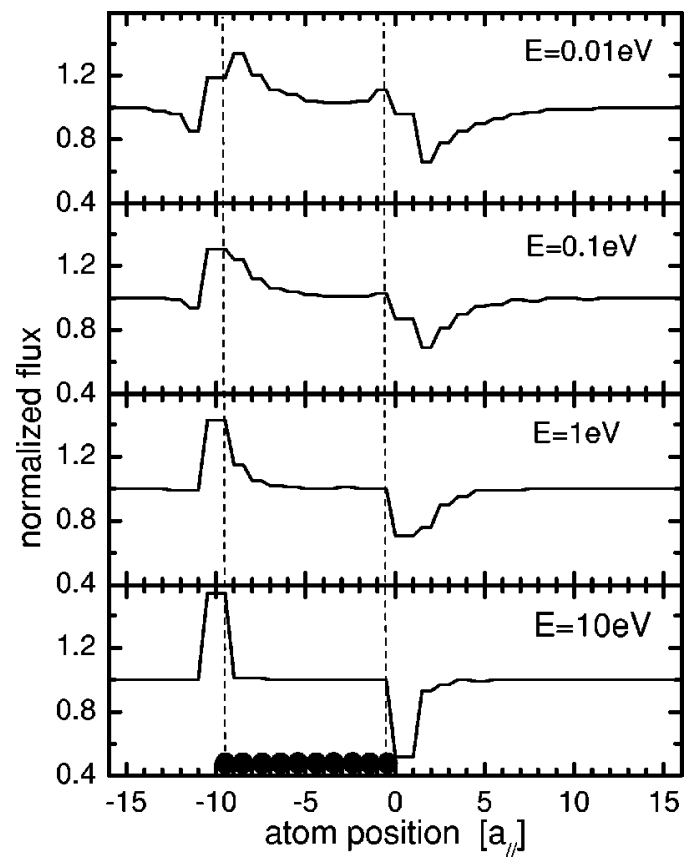

FIG. 5. Flux redistribution for a monolayer island on a $\mathrm{Cu}(001)$ surface as a function of the energy of the incident $\mathrm{Cu}$ atom. The deposition beam is at $50^{\circ}$. The dashed lines indicate the region in which the deposited atoms land on top of the island.

incident beam is thus expected. Although the influence of steering on the morphology at an angle of $50^{\circ}$ was found to be small for low coverages, ${ }^{6}$ the effect on the magnetic properties was already found to be substantial. ${ }^{7,8}$

Although the depleted flux behind the island seems to be independent of energy, in detail there are differences. Figure 5 shows the flux as a function of position on the surface. For the high-energy case a clear shadowing occurs which for this angle of incidence is limited to one adsorption position. For the lower energies, the minimum is less deep and its location shifts away from the island. This is due to the incident atom spending more time above the island and especially by the attraction exerted by the descending step. The descending step in this is defined as the region of about five atoms before and after the actual step. The lateral velocity component can even change its sign in this region. As a result the flux is enhanced on the island at the down step and also in the first deposition position behind the step. The extent of the influence region increases markedly with lower energy. For the high-energy case, the influence region is limited to 2-3 lattice distances, while for the low energies up to seven lattice distances beyond the island down edge. It is therefore remarkable that despite the about three times longer region of influence, the net negative flux behind the island is almost energy independent. At the illuminated step edge the differences for the various energies are clear. For high energies additional flux is deposited just in front of the island as expected from geometry. For the lowest energy, there is hardly any extra flux just in front of the island, even in contrast there is also a depletion of flux. Due to the ascending step of the island the material that would for geometric reasons be deposited in front of the island is now completely deposited

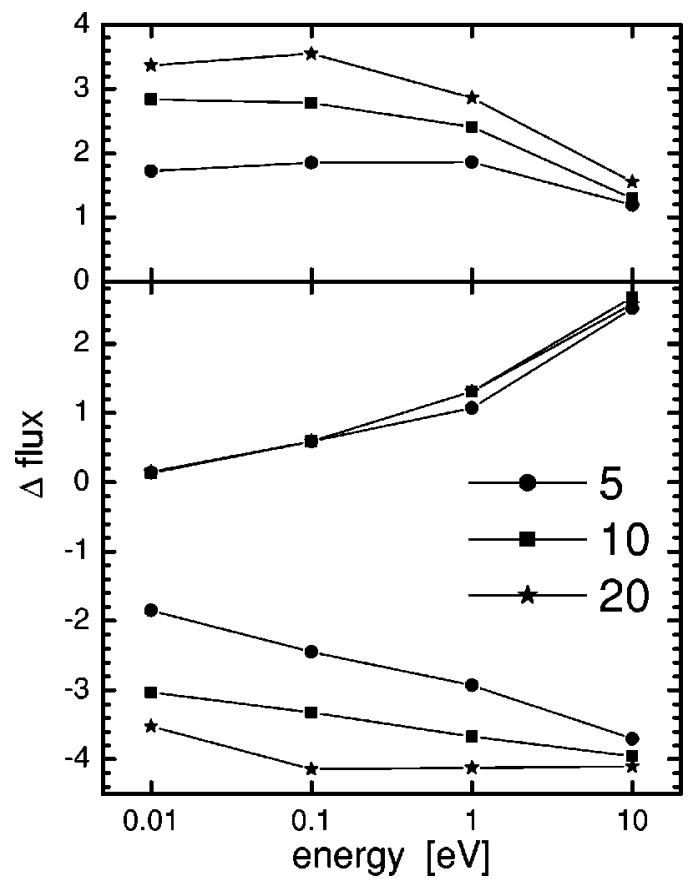

FIG. 6. Flux redistribution for a monolayer island on a $\mathrm{Cu}(001)$ surface as a function of the energy of the incident $\mathrm{Cu}$ atom. The deposition beam is at $80^{\circ}$. Shown are the additional flux on top of the island (upper panel) and the additional flux in front (positive) and behind the island (lower panel). Calculation are depicted for a 5,10 , and 20 atom wide island. The dashed line indicates the geometric shadow value of the decrease in flux behind the island.

on top of especially the first three atoms of the island. This implies that only for island sizes below five atoms an influence of the island size is expected and as for the descending step an ascending step region of a few atoms before and after the actual step can be defined. An additional flux of $18 \%$ per adsorption site occurs for the five atoms wide island, with only 10 and $5 \%$ for, respectively, the 10 and 20 atoms wide island. It depends on especially the Ehrlich-Schwoebel barrier, the nucleation rate and the island size whether this extra flux results in a homogeneous flux over the island edge, and thus to anisotropic islands in growth.

For grazing incidence the flux redistribution clearly becomes atom energy and island size dependent, see Fig. 6. For the 20 atoms large island and all high energy cases the decrease in flux is about equal to the value expected from geometric shadowing. For the small islands the decrease can be only half this value for the low energies. The time of the atom above the island before it is deposited behind the island becomes crucial for the decrease in flux at glancing incidence. The differences with island size does not occur for the deposition in front of the island. At the step up a redistribution dependent on the atom energy takes place, but already an island size of five atoms completely determines this flux redistribution. Similar effects are present as calculated for the $50^{\circ}$ angle of incidence. However, the excess flux on top of the island for this glancing incidence is distributed over the entire island with the accent on the first part, see Fig. 7. The details of the tail of the flux behind the island are striking. The geometric shadow region is four atomic distances 


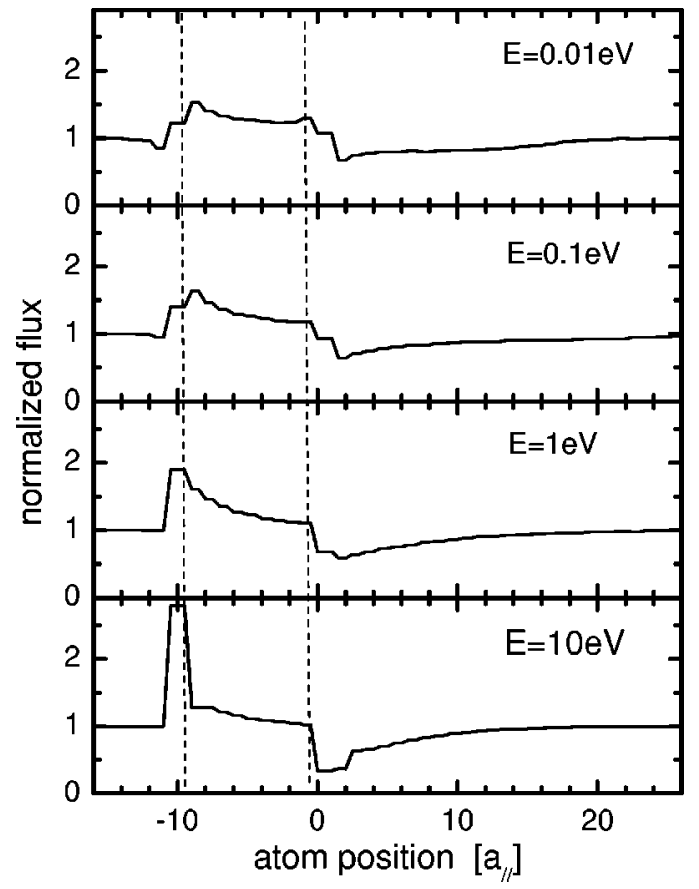

FIG. 7. Flux redistribution for a monolayer island on a $\mathrm{Cu}(001)$ surface as a function of the energy of the incident $\mathrm{Cu}$ atom. The deposition beam is at $80^{\circ}$.

wide and still a marked deposition flux is present in this area for all energies. The region in which the flux is reduced is for all energies considered about 20 atomic distances. This distance is about similar for all three island sizes. For the low energies, the flux becomes more and more homogeneously spread over the island. In total, an additional flux per adsorption site on the island of $35 \%$ for the five atoms wide island to $17 \%$ for the 20 atoms wide island is observed.

\section{THREE LAYER ISLANDS}

The influence of three-dimensional islands on the flux redistribution was simulated with a three ML high island with a base size of 20 atoms and a (115) facet. This implies that next to the terrace and on top of the island, atoms can also be deposited on the small terraces of the (115) facet. The two on either side of the island are denoted by steps 1 and 2, respectively, with a step edge to the terrace and the top of the island, see Fig. 8 for the definition of nomenclature used.

Figure 9 shows the influence at normal incidence. On top of the island a similar additional flux is observed as for the monolayer high island. However, also the step related ter-

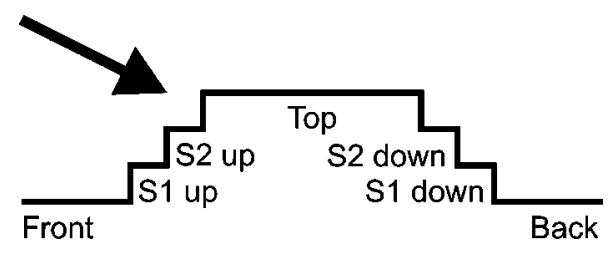

FIG. 8. Definition of the various parts of the three layer island with (115) facets, front, step 1 up, step 2 up, top, step 2 down, step 1 down and back. The incident flux is from the left.

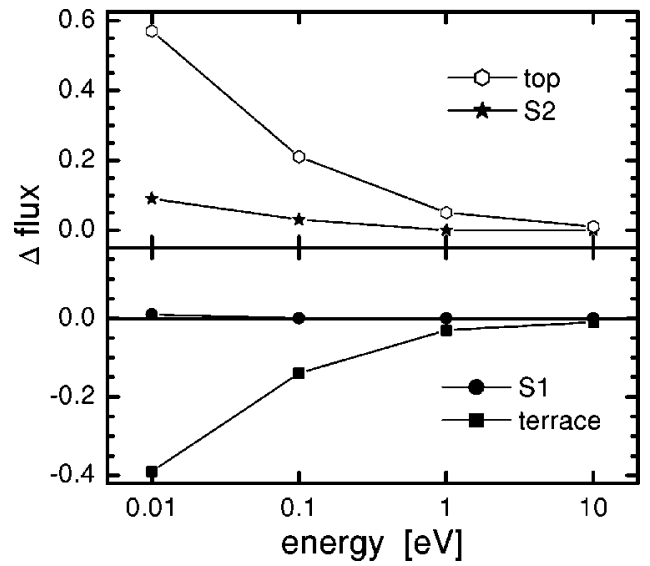

FIG. 9. Flux redistribution for a three layer high island on a $\mathrm{Cu}(001)$ surface as a function of the energy of the incident $\mathrm{Cu}$ atom. The deposition beam is at normal incidence.

races steps 1 and 2 attract additional flux for the low energies. As a result, 1.5 times less flux is deposited on the substrate terrace for the lowest energy.

Figure 10 shows the situation for $50^{\circ}$ angle of incidence. For a monolayer high island it was observed that the influence region for the high-energy case was limited to 2-3 atomic distances behind the step edge. As a result, for the three layers high island an almost similar decrease in flux is observed for steps 1, 2, and the terrace on the shadow side of the island. For lower energies the disturbance by the island clearly results in an attraction towards the top of the island as

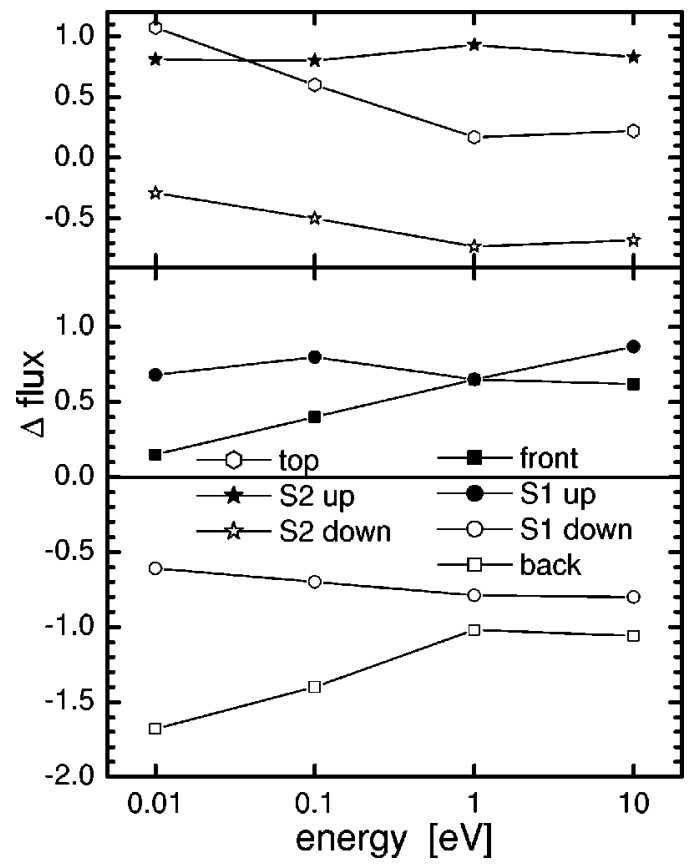

FIG. 10. Flux redistribution for a three layer high island on a $\mathrm{Cu}(001)$ surface as a function of the energy of the incident $\mathrm{Cu}$ atom. The deposition beam is at $50^{\circ}$. Shown are the net flux in front of the terrace, on the first terrace of the facet, the second terrace of the facet, on top and behind the island. Both the illuminated (up) and nonilluminated (down) facet step are shown. 


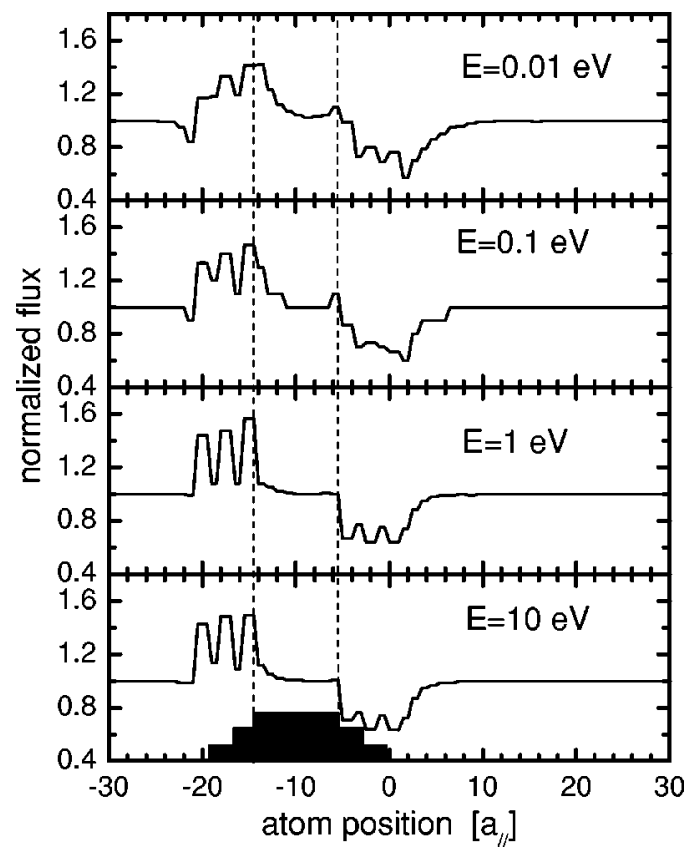

FIG. 11. Flux redistribution for a three layer high island on a $\mathrm{Cu}(001)$ surface as a function of the energy of the incident $\mathrm{Cu}$ atom. The deposition beam is at $50^{\circ}$.

the decrease in flux on the terrace becomes larger, while relatively more material is deposited on step 2 . The sum of the fluxes on step 1, step 2, and the terrace on the shadow side is for all energies about equal. The details of this flux redistribution are depicted in Fig. 11.

The additional flux on the illuminated side shows a relatively similar behavior as the shadow side. For the energies of 1 and $10 \mathrm{eV}$, the additional flux for steps 1,2 , and the illuminated side of the terrace are about equal and the amount similar to the lower flux on the shadow side, i.e., only a small extra flux is deposited on the island. This extra flux is also deposited on the illuminated side of the island top. For lower energies, the additional flux on steps 1 and 2 is hardly changed, while the flux on the illuminated terrace is reduced in favor of a deposition on top. The additional flux on the island is similar to the monolayer island, with the exception that in this case also for $10 \mathrm{eV}$ still an increase in flux is observed. This can be especially relevant for the growth of columnar structures as observed oblique sputter deposition. Also the additional flux on steps 1 and 2 will lead to an asymmetric shape of the island, with a steeper facet on the illuminated side.

The flux redistribution for $80^{\circ}$ incidence is depicted in Fig. 12. In contrast to the $50^{\circ}$ situation, the net fluxes on steps 1,2 , and the terrace on the shadow side are no longer equal for the higher energies. As already the geometric shadow length is larger than the length of the facet terrace, depletion of flux on especially the terrace in the shadow occurs. The geometric shadow length is $12 a_{/ /}$, while a detailed analysis as shown in Fig. 13 shows that independent of energy up to $26 a_{/ /}$behind the end of the upper terrace of the 3D island a flux deficiency is noticeable. The total flux deficiency on the shadow side is, however, strongly dependent

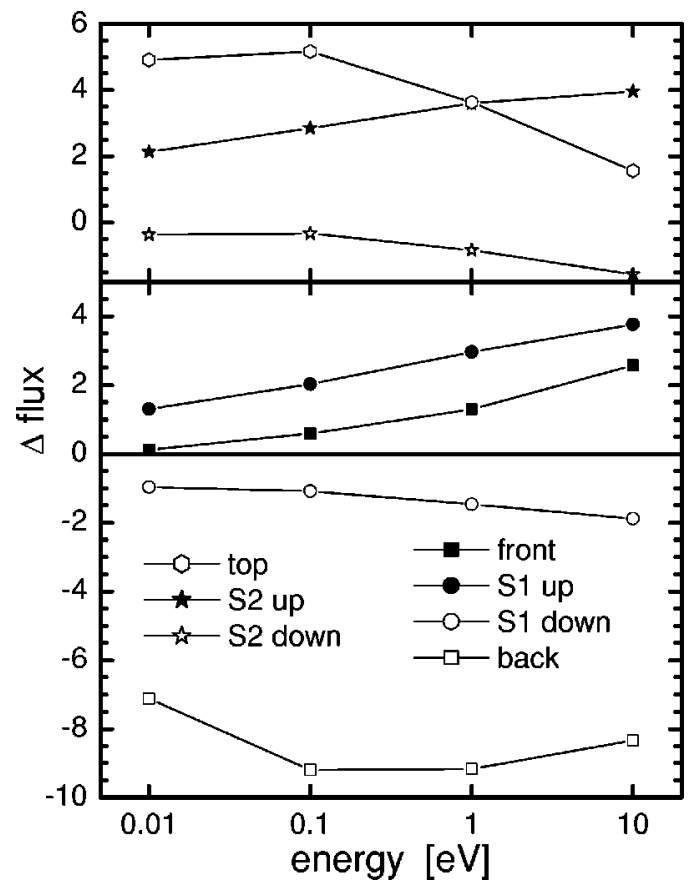

FIG. 12. Flux redistribution for a three layer high island on a $\mathrm{Cu}(001)$ surface as a function of the energy of the incident $\mathrm{Cu}$ atom. The deposition beam is at $80^{\circ}$. Shown are the net flux in front of the terrace, on the first terrace of the facet, the second terrace of the facet, on top and behind the island. Both the illuminated (up) and nonilluminated (down) facet step are shown.

on particle energy, as for $1 \mathrm{eV}$ particles a total of -11.6 $\times F$ incident atoms are redistributed, while for $0.01 \mathrm{eV}$ this number is $-8.6 \times F$. The homogeneous incidence flux $F$ is, as before, set at a rate of 1 particle per $a_{/ /}$per unit of time. This situation is similar to the one observed for the small monolayer high islands, see Fig. 6 .

The saturation of the on top deposited flux for the lower energies is similar to the one observed for the monolayer high islands, with $20 \%$ additional flux. The illuminated facet shows a markedly enhanced steering behavior compared to the $50^{\circ}$ situation. The terrace in front of the facet hardly sees any additional flux, while step 1 has $1.2 \times F$ atoms extra deposited, i.e., almost a doubling of the local deposition rate. However this increase is almost tripled for step 2 to which $2.1 \times F$ additional atoms are drawn to. This shows that for low-energy particles, the apex on the illuminated side acts as a strong attractor and an increase in facet angle just below the apex can be expected. The high energy situation also illustrates the onset for the well known columnar growth in sputter deposition. With increasing thickness the center of mass of every deposited layer moves towards the illumination source. At these incidence angles the illuminated facet will tend to a (111) orientation. ${ }^{6}$ The preference for the apex, as observed most strongly for the lower energies, may finally even result in the formation of overhangs. ${ }^{12}$ Especially for the combination of high fluxes and low temperatures, stable nuclei can be created on these (111) oriented facets. These protrusions in turn will act as flux attractors in further stages of growth.

The shadow region observed will influence the growth 


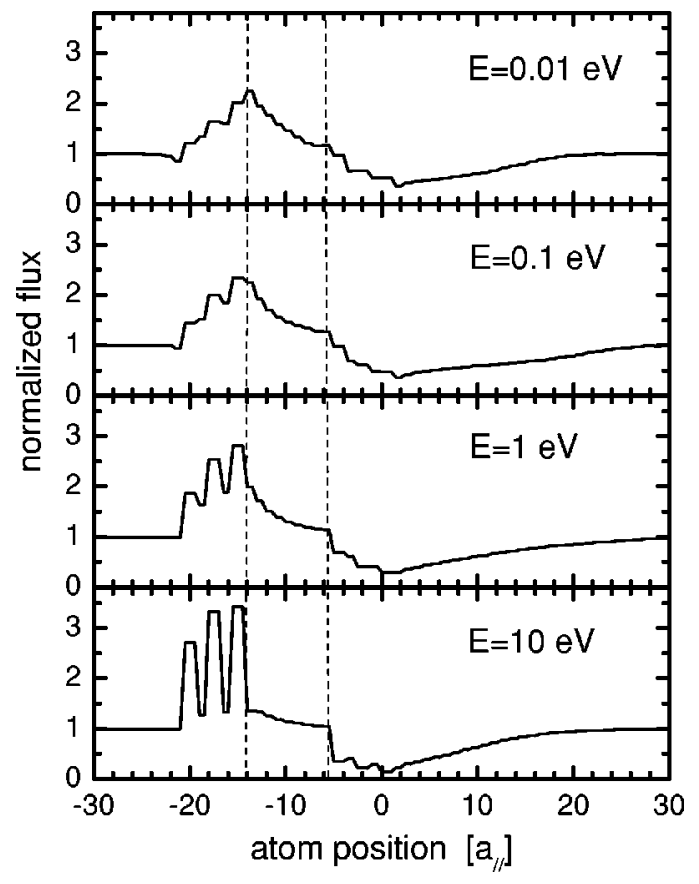

FIG. 13. Flux redistribution for a three layer high island on a $\mathrm{Cu}(001)$ surface as a function of the energy of the incident $\mathrm{Cu}$ atom. The deposition beam is at $80^{\circ}$.

further when the distance between islands is similar or smaller than the shadow length. Such a situation occurs for the three layer $\mathrm{Cu}(001)$ island for deposition temperatures below $250 \mathrm{~K}$ and a flux of $0.2 \mathrm{ML} / \mathrm{min}$. With increasing island thickness, the shadow region will extend and thus become increasingly important for glancing incidence growth.

\section{DISCUSSION OF THE MODEL LIMITATIONS}

For a qualitative estimate of the consequences of steering in a wide range of atom deposition geometries and kinetic energies, we have chosen to run deterministic classical trajectory calculations, using a pairwise additive Lennard Jones potential. Moreover, we assume a rigid lattice for the substrate. Obviously we are well aware of the shortcomings of this approach, which are manifold. First the potential is certainly defective for the description of the metal-atom-metalsurface interaction. However, a comparison with the computed results in Ref. 2 shows that the trajectories are pretty well represented (see also Sec. II). In support of our approach, we also note that it adequately provides a qualitative description of the experimentally observed enhancement of kinetic roughening at grazing incidence deposition compared to normal incidence deposition. In addition, our approach is believed to mimic qualitatively well the morphology induced magnetic properties of films sputter deposited at grazing incidence. ${ }^{15}$ It also allows a quick assessment of the steering features for grazing incidence deposition, which would require a nonpractical, very large cell in exact molecular dy- namic calculations. We do note that the forces exerted by the arriving atoms on the substrate atoms may give rise to appreciable movement of the lattice atoms, especially for those with lower coordination numbers. In general, these features will lead to stronger steering than calculated here. Finally, we emphasize that by its nature our consideration completely fails to describe phenomena due to the actual impact. Especially again for the involved low coordinated impact sites there may well be significant deviations from incorporation of atoms in a real lattice. So finally for a quantitative description of steering exact calculations should be made. However, the observed trends are clear and we are confident that they provide a solid and relevant, though qualitative indication of the features to be expected for steering of atoms arriving at various kinetic energies and polar angles of incidence. We expect that our approach probably under-estimates the strength of steering, especially for very small adatom features on the surface.

\section{CONCLUSION}

At normal incidence, the interaction through long range van der Waals forces between an incoming particle and a substrate is important only for low particle kinetic energies $(0.01 \mathrm{eV})$. Note, however, that in the presence of very high protrusions (not considered here), arriving atoms will be deflected at much higher energies. Also the size of structures on the substrate is for an angle of incidence of $50^{\circ}$ as for normal incidence not an important parameter. For high particle energies $(>1 \mathrm{eV})$ as, e.g., relevant for sputter deposition, the flux redistribution is insignificant and already the geometric shadow limit is observed. However, for lower particle energies a marked redistribution of flux is calculated. Additional flux is deposited on top of the structure, both for a monolayer high island and the three layer high island. The shadow region extends over a much larger region than the geometric shadow region, but the amount of involved material is about equal to the reduced flux from geometric considerations. For grazing incidence deposition a strong focussing on the illuminated apex is observed for the low particle energies. This results in a massive shift of flux from just in front of the island to on top of the island. Also a depletion region just before the step is observed. For higher islands, an even larger focussing of the flux on the top of the island for low particle energies can be expected. These particle energies, present in the low velocity range of a thermal evaporation source are very sensitive to local disturbances, clearly observed at the down steps, where the lateral velocity changes sign, and extra material is deposited just in front of the down step. The start of columnar growth and the formation of overhangs is quite likely in these situations.

\section{ACKNOWLEDGMENTS}

B.P. wishes to acknowledge fruitful private discussions with Art Voter and Francesco Montalenti. 
*Electronic address: h.wormeester@tn.utwente.nl

${ }^{1}$ N.J. Shevchik, J. Non-Cryst. Solids 12, 141 (1973).

${ }^{2}$ D.E. Sanders and A.E. DePristo, Surf. Sci. 254, 341 (1991).

${ }^{3}$ D.E. Sanders, D.M. Halstead, and A.E. DePristo, J. Vac. Sci. Technol. A 10, 1986 (1992).

${ }^{4}$ W.D. Luedtke and U. Landman, Phys. Rev. B 40, 11733 (1989).

${ }^{5}$ S. van Dijken, L.C. Jorritsma, and B. Poelsema, Phys. Rev. Lett. 82, 4038 (1999).

${ }^{6}$ S. van Dijken, L.C. Jorritsma, and B. Poelsema, Phys. Rev. B 61, 14047 (2000).

${ }^{7}$ S. van Dijken, G. Di Santo, and B. Poelsema, Appl. Phys. Lett. 77, 2030 (2000).
${ }^{8}$ S. van Dijken, G. Di Santo, and B. Poelsema, Phys. Rev. B 63, 104431 (2001).

${ }^{9}$ M. Raible, S.G. Mayr, S.J. Lint, M. Moske, P. Hanggi, and K. Samwer, Europhys. Lett. 50, 61 (2000).

${ }^{10}$ M. Raible, S.J. Lint, and P. Hanggi, Phys. Rev. E 62, 1691 (2000).

${ }^{11}$ J. K. Seo, S. M. Kwon, and J. S. Kim (unpublished).

${ }^{12}$ F. Montalenti and A.F. Voter, Phys. Rev. B 64, 081401 (2001).

${ }^{13}$ F. Montalenti, M.R. Sörensen, and A.F. Voter, Phys. Rev. Lett. 87, 126101 (2001).

${ }^{14}$ D. P. Jackson, Interatomic Potentials and Simulations of Lattice Defects (Plenum, New York, 1972).

${ }^{15}$ J. C. Lodder (private communication). 